\title{
Molecular detection and pathogenicity of a nucleopolyhedrovirus isolated from looper caterpillar (Hyposidra talaca), a tea pest
}

\author{
Soma Dasgupta ${ }^{1,4} \cdot$ Hijam Ranjit Singh $^{2} \cdot{\text { Sudripta } \text { Das }^{2,5} \cdot \text { Sunil Kumar Pathak }}^{3}$ • \\ Rakesh Kumar Bhola ${ }^{4}$
}

Received: 18 August 2016/Accepted: 8 November 2016/Published online: 16 November 2016

(C) The Author(s) 2016. This article is published with open access at Springerlink.com

\begin{abstract}
Hyposidra talaca is a major defoliating pest of tea plants in north-eastern part of India. In this study, we look for variations (if any) in the attacking virus. Viral samples were collected from different regions of the northern part of West Bengal in India and were analyzed by PCR technique to study the variations across the region. The partial segment of the HytaNPV polyhedrin gene was cloned and sequenced. A $527 \mathrm{bp}$ nucleotide sequence containing highly conserved region from polyhedrin gene of HytaNPV was observed. The blast homology search for studied polyhedrin gene showed $98 \%$ sequence identity
\end{abstract}

Soma Dasgupta

soma.dasgupto@gmail.com

Hijam Ranjit Singh

jit_eini@yahoo.co.in

Sudripta Das

sudriptadas@gmail.com

Sunil Kumar Pathak

skpathak3@gmail.com

Rakesh Kumar Bhola

bhola_zoology@yahoo.co.in

1 Plant Protection Department, Tea Research Association, North Bengal Regional Research and Development Centre, Nagrakata, Jalpaiguri 735225, West Bengal, India

2 Department of Biotechnology, Tea Research Association, Tocklai Tea Research Institute, Jorhat, Assam 785008, India

3 Advisory Services of Assam, Tea Research Association, Tocklai Tea Research Institute, Jorhat, Assam 785008, India

4 Department of Zoology, Gauhati University, Guwahati 781014, India

5 Present Address: Department of Medicinal Plant, Institute of Bioresources and Sustainable Development (IBSD), Imphal, Manipur 795004, India with the sequence of previous reported NPV of H. talaca, H. infixaria and Buzura suppressaria. Pathogenicity study against second instar $H$. talaca indicated that the $\mathrm{LC}_{50}$ values ranged from $4.61 \times 10^{5}$ to $7.57 \times 10^{5}$ polyhedral occlusion bodies per $\mathrm{ml}(\mathrm{POBs} / \mathrm{ml})$ and the $\mathrm{LT}_{50}$ values ranged from 4.2 to 6.66 days. Sequencing result reveals that the same HytaNPV strain dominates across this area and the pathogenicity indicates its potential as an alternative to chemical insecticides to control $H$. talaca.

Keywords Hyposidra talaca · Nucleopolyhedrovirus · Polyhedrin gene $\cdot$ PCR $\cdot$ Bioactivity

\section{Introduction}

Baculoviruses are renowned for most widely studied virus of insects. More than 700 baculoviruses have been isolated mainly from the insect species of the orders Diptera, Hymenoptera and Lepidoptera. Nucleopolyhedroviruses (NPVs), which are a part of baculovirus, are considered as potential biocontrol agents and have been applied successfully against larvae of many insect pests in the world (Hu et al. 1993; Ma et al. 2007). NPVs are mainly pathogenic for insects of Lepidoptera. Polyhedrin gene of NPV is considered as one of the most conserved baculovirus genes (Jehle 2004), and for the development of the genetic amplification technique, this gene proved its efficiency (Woo 2001). Polymerase chain reaction (PCR) technique can amplify target DNA sequence and it has been used in baculovirus screening since the last three decades (Burand et al. 1992; Ikuno et al. 2004; Galal 2009; Hewson et al. 2011; Arneodo et al. 2012). The PCR technique is suitable for diagnosis of viruses as identification, 
characterization as well as for their genome investigation (Moraes and Maruniak 1997; Fattouch et al. 2005).

The characteristics of NPVs that empower it to be useful as biopesticide are its high pathogenicity, host specificity, less chances of cross-infectivity and having the ability of rapid distribution. The virus has stable occlusion bodies and can effectively suppress a target pest from a crop field (Moscardi 1999). In this regard, Hyposidra talaca nucleopolyhedrovirus (HytaNPV) is an extremely infectious natural agent causing most destructive disease to the tea pest Hyposidra talaca. In India, HytaNPV is reported in Terai and Dooars part of North Bengal (Mukhopadhyay et al. 2010; Antony et al. 2011; Sinu et al. 2015). The development of such control agent starts with the isolation of naturally occurring entomopathogens and followed by their identification, characterization as well as the evaluation of their virulence.

The H. talaca looper caterpillar (Walk.) (Lepidoptera: Geometridae) is an economically significant defoliating pest of the tea belt in the north-eastern part of India. Previously, Buzura suppressaria (Guen.) (Lepidoptera: Geometridae), a major tea defoliator in India and China, was dominant in this region, but gradually it was suppressed by the current destructive pest $H$. talaca (Hazarika et al. 2009). In the last decade, H. talaca was introduced into tea agro-ecosystem and caused severe damages in the tea industry. It spread all over the northern part of West Bengal (WB) and Assam in 2006 (Sinu et al. 2011). As the management of $H$. talaca is carried out with their population monitoring and respective damage control application of chemical pesticides, which are mainly organophosphates and synthetic pyrethroids (Sannigrahi and Talukdar 2003; Sarker and Mukhopadhyay 2006), there is an urgent need to find an alternative control method. Among few natural enemies of the pest, HytaNPV is found to be active and causes epizootics within a dense population of $H$. talaca (Sinu et al. 2015). Successive generations of insect host are infected by polyhedra; thus the host serves as a reservoir of inoculums.

Due to the high potency of the virus as chemical alternative, finding out the naturally improved isolates of the virus is a matter of great interest. PCR-based diagnosis was used for identification, establishment of relationship and also to find out the variation within the tested HytaNPVs. The primary aim of the study was to identify the most potential isolate of HytaNPV. Examination of the samples from four different geographic locations was done for the above-mentioned identification and simultaneously infectivity evaluation was conducted to determine their successful practical use, by laboratory bioassay. This study may play an important role in the programs of integrated pest management (IPM).

\section{Materials and methods}

\section{Larval rearing}

To investigate the sensitivity of $H$. talaca, the second instar larvae of this looper caterpillar were originally collected from Dooars region, West Bengal, India. The collection site was free of pesticides since 6 months and the larvae were carried with leaves to reduce their transportation disturbances. They were reared in the laboratory of the Plant Protection Department, North Bengal Regional Research and Development Centre, Tea Research Association (TRA), for three successive generations at $28 \pm 2{ }^{\circ} \mathrm{C}, 72 \pm 3 \%$ relative humidity and a $13 \mathrm{~L}: 11 \mathrm{D}$ photoperiod. Fresh tea leaves were provided as food after sterilizing with $10 \%$ formalin for $5 \mathrm{~min}$ and rinsed with sterile double-distilled water. These larvae were used for viral propagation and bioassays. The larvae were also examined daily to eliminate the secondary infection.

\section{Viruses}

The HytaNPV samples, used in the PCR study, were collected during 2012-2013 from geographically distinct localities covering eastern Dooars, western Dooars, central Dooars and Terai region of West Bengal, India. The average rainfall of Terai and Dooars are approximately 3500 and $3160 \mathrm{~mm}$, respectively, situated between $26^{\circ} 16^{\prime}$ and $27^{\circ} 12^{\prime}$ north latitudes and $87^{\circ} 59^{\prime}$ and $89^{\circ} 53^{\prime}$ east longitudes. The virus samples were collected from ten tea estates located across these four distinct geographic regions (Table 1). From each collection site, 50-60 virus-infected larvae were sampled. The virus-infected larvae were collected as samples considering the primary signs of typical NPV infection in larval stage like flaccidity, rupturing of the cuticle and hanging upside down by their abdominal legs. Later, the presence of HytaNPV was ascertained by microscopic view and PCR study. The individual larva was collected in $1.5 \mathrm{ml}$ centrifuge tube and the collected samples were kept at $-20{ }^{\circ} \mathrm{C}$ for future use.

\section{Purification of HytaNPV}

For molecular analysis, each of aqueous homogenate of viral samples containing polyhedral occlusion bodies (POBs) was purified by two rounds of centrifugation. The putrefied samples were homogenized with pestle and mortar, and the concentrates were diluted with sterile double-distilled water. The crude filtrate was initially centrifuged for $5 \mathrm{~min}$ at $600 \mathrm{rpm}$ to remove larger contaminants. The supernatant was collected in a new centrifuge tube for further centrifugation at $8000 \mathrm{rpm}$ for 
Table 1 Details of the survey site location

\begin{tabular}{llll}
\hline Region & Survey site & Isolate referred as & Geographic coordinate \\
\hline Western dooars & Kumlai tea estate & KML & $26^{\circ} 50^{\prime} \mathrm{N}, 88^{\circ} 41^{\prime} \mathrm{E}$ \\
& Washabari tea estate & WSB & $26^{\circ} 52^{\prime} \mathrm{N}, 88^{\circ} 32^{\prime} \mathrm{E}$ \\
Central dooars & Bhagatpur tea estate & BGP & $26^{\circ} 53^{\prime} \mathrm{N}, 88^{\circ} 55^{\prime} \mathrm{E}$ \\
& Ambari tea estate & AMB & $26^{\circ} 52^{\prime} \mathrm{N}, 89^{\circ} 03^{\prime} \mathrm{E}$ \\
& Karbala tea estate & KRB & $26^{\circ} 47^{\prime} \mathrm{N}, 89^{\circ} 04^{\prime} \mathrm{E}$ \\
& Telipara tea estate & TLP & $26^{\circ} 43^{\prime} \mathrm{N}, 89^{\circ} 03^{\prime} \mathrm{E}$ \\
& Bara Dighi tea estate & BRD & $26^{\circ} 47^{\prime} \mathrm{N}, 88^{\circ} 46^{\prime} \mathrm{E}$ \\
Eastern dooars & Lakhipara tea estate & LKP & $26^{\circ} 49^{\prime} \mathrm{N}, 89^{\circ} 00^{\prime} \mathrm{E}$ \\
Terai & Rajabhat tea estate & RJB & $26^{\circ} 39^{\prime} \mathrm{N}, 89^{\circ} 29^{\prime} \mathrm{E}$ \\
\hline
\end{tabular}

20 min to pellet the virus and then washed with sterile double-distilled water for three times. The pellets were finally dissolved in $1 \mathrm{ml}$ sterile double-distilled water and conserved at $-20{ }^{\circ} \mathrm{C}$. The quantification of POBs was carried out by using Neubauer hemocytometer (Marienfeld, Germany) under phase-contrast microscope (Olympus BX 51). For bioassays, the viral samples were purified by following the same method.

\section{Virus propagation}

Four HytaNPV isolates (SMB, RJB, WSB and TLP), belonging to four different geographic regions, were taken for bioassay tests and multiplied in second instar $H$. talaca larvae for mass propagation. In case of each isolate, the multiplication of HytaNPV was conducted by isolation of POBs from infected cadavers. For multiplication of each isolate, 150 larvae were fed on tea leaves dipped with virus suspension in a concentration of $10^{7} \mathrm{POBs} / \mathrm{ml}$. After $2 \mathrm{~h}$ of starvation, they were exposed to virus-contaminated leaves for $24 \mathrm{~h}$. Virus-induced larvae were kept as 30 larvae per group and reared in the laboratory at $28 \pm 2{ }^{\circ} \mathrm{C}, 72 \pm 3 \%$ relative humidity and a $13 \mathrm{~L}: 11 \mathrm{D}$ photoperiod. Microscopic view confirmed the presence of POBs in the cadavers of virus-induced larvae.

\section{Viral DNA extraction and PCR amplification}

After following the purification method, viral DNA was extracted from POBs of each of ten field collected HytaNPV samples and purified with QIAamp DNA Mini Kit (Qiagen) according to the manufacturer's protocol. The final DNA was obtained after cleaning several times with ethanol and diluted wash solution. Later, DNA was eluted and re-suspended in $20 \mu \mathrm{l}$ molecular-grade water (Himedia). The isolation of DNA was confirmed by electrophoresis in $1 \%$ agarose gel and quantified with BioPhotometer (Eppendorf).

A highly conserved region of polyhedrin gene from HytaNPV isolates was amplified. The PCR was performed using the degenerate primer (F: 5'-GGACCSGGYAARA AYCAA AAA-3' and R: 5'-GCRTCWGGYGCAAAYT CYTT- $3^{\prime}$ ) designed according to Antony et al. (2011). The PCR reaction was carried out taking 50-100 ng of viral DNA in a $25 \mu \mathrm{l}$ reaction solution containing $1 \mathrm{X}$ PCR buffer (Invitrogen, USA), $1.5 \mathrm{mM} \mathrm{MgCl}_{2}$ (Invitrogen, USA), $0.5 \mathrm{mM}$ dNTPs (Bangalore Genei, India), $1 \mathrm{U}$ Platinum Taq DNA polymerase (Invitrogen, USA) and $0.5 \mu \mathrm{M}$ of each primer. PCR amplification was performed in a DNA thermal cycler (Veriti Thermal Cycler, Applied Biosystems, CA, USA). The conditions used were initially denaturation for one cycle at $94{ }^{\circ} \mathrm{C}$ for $5 \mathrm{~min}$, followed by 35 repeated cycles of $94{ }^{\circ} \mathrm{C}$ for $30 \mathrm{~s}, 50{ }^{\circ} \mathrm{C}$ for $30 \mathrm{~s}, 72{ }^{\circ} \mathrm{C}$ for $30 \mathrm{~s}$, and the final extension cycle at $72{ }^{\circ} \mathrm{C}$ for $7 \mathrm{~min}$. The amplified products were resolved in $1.5 \%$ agarose gel stained with ethidium bromide.

\section{Cloning and sequencing of the polyhedrin gene}

The positive PCR product of the SMB isolate was eluted from the gel using HiPura Agarose Gel DNA Purification Spin Kit (Himedia, India) following the manufacturer's protocol. The eluent was ligated into the pGEM-T vector (Promega, UK) in a 3:1 (insert: vector) molar ratio with $\mathrm{T}_{4}$ DNA ligase as described by the manufacturer. The ligated products were transformed into Escherichia coli DH10 $\beta$ competent cells (Invitrogen, USA). Following amplification of recombinant clone with M13 universal forward and reverse primers, the sequencing was performed using the BigDye Terminator v1.0 cycle sequencing kit (Applied Biosystems) in ABI 3500 Genetic Analyzer (Applied Biosystems). 


\section{Sequence comparison and phylogenetic analysis}

The sequence data were assembled into contig by DNA Dragon Version 1.5.6 (SequentiX, Germany) followed by the multiple-sequence alignments construction with the highly similar DNA and protein sequences using the Clustal W program (Thompson et al. 1994). The obtained nucleotide sequence was blasted to the GenBank database (blastx) to retrieve similar nucleotide and amino acid sequences, which were used for phylogenetic tree construction. The phylogenetic tree of aligned amino acid sequences was generated by the neighbor-joining (NJ) algorithm (Saitou and Nei 1987) using Molecular Evolutionary Genetics Analysis (MEGA) v6.06 (Tamura et al. 2013). To estimate the confidence limits of nodes, 1000 bootstrap samples were generated. To reduce the impact of partial sequences of polyhedrin available in the GenBank database and to maximize the use of the available information, the pairwise comparison of amino acid sequences was performed by ClustalW with the default parameters.

\section{Bioassays}

The biological activity of four HytaNPV isolates, viz., SMB, RJB, WSB and TLP (belonging to four different geographic regions), was tested against early second instar H. talaca larvae. Bioassay tests were conducted using purified viral suspension by leaf-dip feeding technique. The virus concentrations were quantified with a phase-contrast microscope and a Neubauer hemocytometer. The concentrations of each tested isolates were prepared from the following stock concentrations in $\mathrm{POB} / \mathrm{ml}: 3.6 \times 10^{9}$, $5.7 \times 10^{9}, 2.9 \times 10^{9}$ and $8.1 \times 10^{9}$ for SMB, RJB, WSB and TLP isolates, respectively. The experiments were performed using six concentrations of each virus isolate containing $1 \times 10^{3}, 1 \times 10^{4}, 1 \times 10^{5}, 1 \times 10^{6} 1 \times 10^{7}$ and $1 \times 10^{8} \mathrm{POBs} / \mathrm{ml}$. Larvae were taken from the virusfree rearing culture and starved for $2 \mathrm{~h}$ before feeding viruses. Leaves were dipped in viral suspension, air-dried and fed to larvae for $24 \mathrm{~h}$ prior to feeding on fresh foliages until death or pupation. In the control treatment, the virus suspension was replaced by double-distilled water. For each isolate, three replicates each of 30 larvae were used for each virus concentration and three replicates (30 larvae per replication) of double-distilled water-treated leaves served as control. Larvae were incubated at $27 \pm 1{ }^{\circ} \mathrm{C}$, $72 \pm 3 \% \mathrm{RH}$ and a 13L:11D photoperiod. Virus-induced cumulative mortality was recorded daily till death and the mortality response data were analyzed on the basis of mortality on day 7 post-inoculation. The diseased cadavers were collected and kept in $-20{ }^{\circ} \mathrm{C}$ for further analysis. Later virus infections were confirmed by the presence of
POBs in the cadavers, when viewed under phase-contrast microscopy.

\section{Statistical analysis}

The mortality of larvae was tabulated daily for all NPV isolates vs. dose combinations $(4 \times 6)$. Probit analysis was performed using IBM SPSS release 23.0.0.0 on the basis of mortality data obtained after 7 days of post-inoculation. The median lethal concentrations $\left(\mathrm{LC}_{50}\right)$ for second instar were obtained from the SPSS probit model. Median lethal time $\left(\mathrm{LT}_{50}\right)$ was also determined for each concentration using the equation (Biever and Hostetter 1971):

$\mathrm{LT}_{50}=a+e(c-b) / D$

where ' $a$ ' is the number of hours from the initiation of the test until the reading made immediately before the $50 \%$ mortality was recorded; ' $b$ ' is the total number of larvae dead at the reading immediately before the $50 \%$ mortality was recorded; ' $c$ ' is the $50 \%$ of the total number tested (in our case, it is 45 ); ' $D$ ' is the number of larvae dying in $24 \mathrm{~h}$ during which the $50 \%$ mortality was reached; and ' $e$ ' is the number of hours between mortality counts ( $24 \mathrm{~h}$ in this case).

\section{Results}

\section{Field survey}

The virus-infected larvae were collected from different geographic locations (Dooars and Terai) by primary observation of typical NPV signs and subjected to further studies (Fig. 1). The virus was diagnosed by microscopic study, propagated in $H$. talaca laboratory colony, purified and kept at $-20{ }^{\circ} \mathrm{C}$ for future studies. The number of polyhedral occlusion bodies was calculated by a hemocytometer showing the presence of approximately $8.7 \times 10^{9} \mathrm{POBs} / \mathrm{ml}$ of cadaver.

\section{PCR amplification and sequencing}

The degenerate primer set was used to amplify the targeted sequence from the isolated DNA of the purified polyhedra and successful amplification of the specific polyhedrin gene region of ten isolates of HytaNPV was performed. Amplification was not obtained from the extract of control larvae. The bands obtained from the PCR products of all ten isolates were identical (Fig. 2). Hence, the sequencing was performed by taking only the SMB isolate of the Terai region; then it was compared and cross validated with previously reported HytaNPV (from Dooars region). Hereafter, the studied Terai isolate is denoted as HytaNPV$P$. The amplified product of the concerned isolate showed a 
Fig. 1 a Tea leaves affected by Hyposidra talaca. b Cadaver of Hyposidra talaca showing symptoms of nucleopolyhedrovirus infection

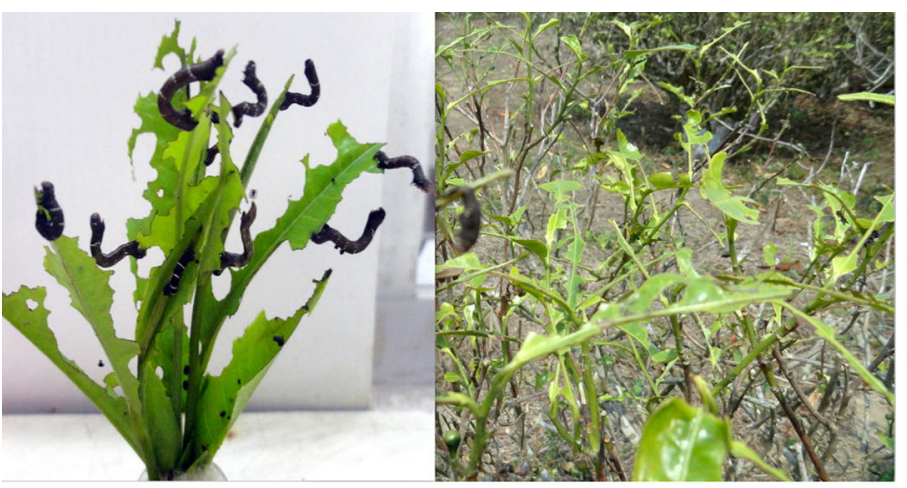

A

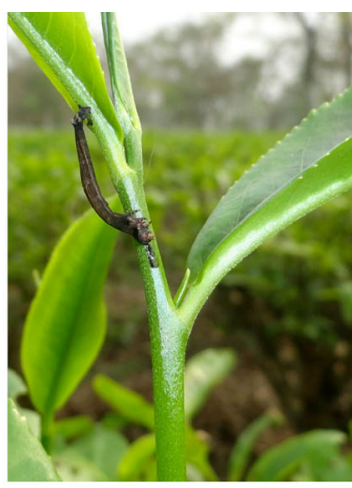

B
Fig. 2 Agarose gel electrophoresis of specific PCRamplified products from ten HytaNPV isolates. Lanes M 1 and M 2, 100 bp Ladder DNA marker (Genei, Bangalore); Lane L1: SMB, L2: LKP, L3: KRB, L4: TLP, L5: KML, L6: WSB, L7: BRD, L8: RJB, L9: BGP and L10: AMB isolate, respectively. The size of the band is shown in bp (base pair)

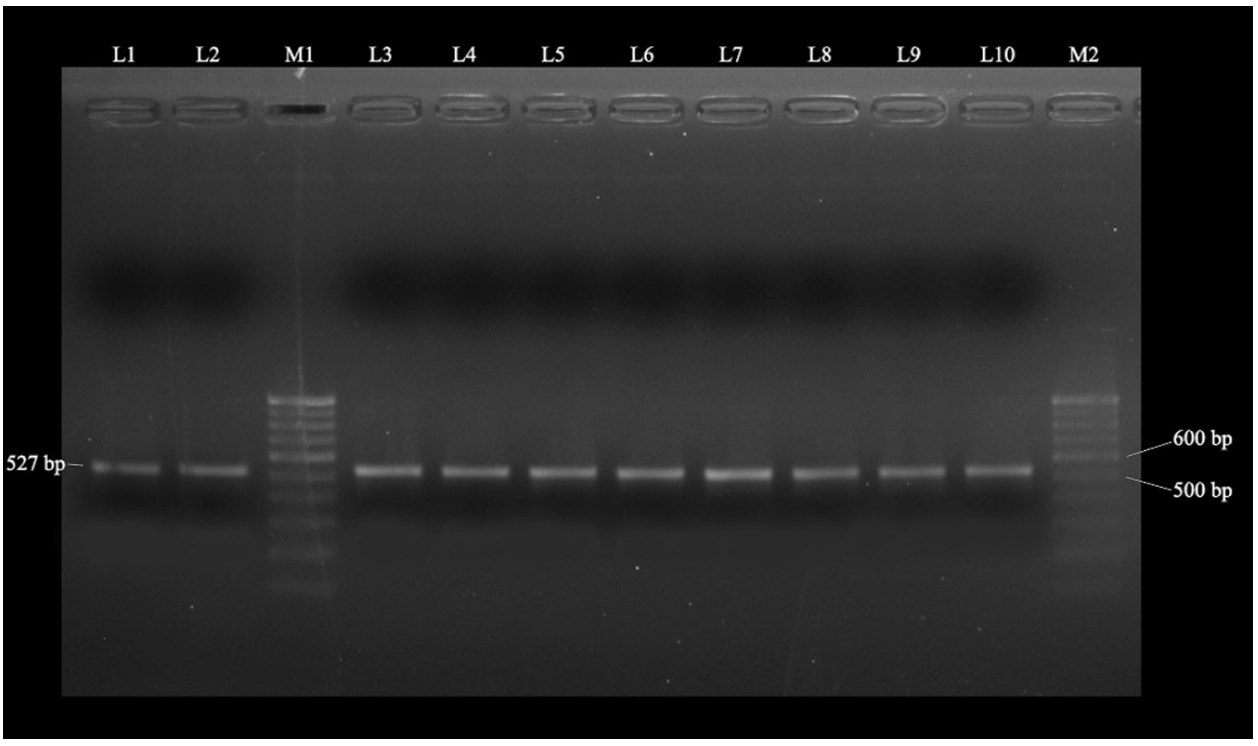

nucleotide sequence of 527 bp (GenBank accession no. KP027542). The deduced protein consists of 175 amino acids with the predicted molecular mass of $19.52 \mathrm{kDa}$.

The obtained nucleotide sequence was blasted in the NCBI GenBank database, the NPV polyhedrin gene was identified and the blastn algorithm indicated the significant homology with 29 NPV isolates. The blast results for the polyhedrin gene of HytaNPV-P (acc. no. KP027542) at the nucleotide level revealed the highest homology of $98 \%$ with the polyhedrin gene of HytaNPV (acc. no. JF510035), $H$. infixaria NPV (HyinNPV, acc. no. JF510036) and $B$. suppressaria NPV (BusuNPV, acc. no. JF510034) previously reported by Antony et al. (2011) and the lowest sequence identity of $79 \%$ was observed in Plusia orichalcea NPV (acc. no. AF019882). On comparing, the multiple-sequence alignment of nucleotide was generated with HytaNPV-P, HytaNPV, HyinNPV and BusuNPV of Indian and Chinese isolate (Fig. 3). In comparison with previously reported HytaNPV, it exhibited ten nucleotides changes (at $6,12,15,36,102,174,192,513,519$ and 522 positions) throughout the DNA segment.
The deduced amino acid sequence was compared to all other reported polyhedrins in the GenBank database, and HytaNPV-P polyhedrin (acc. no. AJN00735) showed $87-100 \%$ homology with other lepidopteran NPVs. The results showed $100 \%$ sequence homology with HytaNPV (acc. no. AEK86285), HyinNPV(acc. no. AEK86286) and BusuNPV (acc. no. AEK86284). In spite of the above similarities, it created $98 \%$ sequence identity with the Chinese isolate of BusuNPV (acc. no. YP009001778), 97\% sequence identity with Ectropis obliqua NPV (EcobNPV, acc. no. YP874194; also reported from China), and Hemileuca sp. NPV (acc. no. YP008378217), and 95\% sequence identity with Helicoverpa armigera NPV (acc. no. ABW06597) and Mamestra configurata NPV (acc. no. NP613084). The lowest sequence identity of $88 \%$ was observed with Bombyx mori polyhedrin (acc. no. NP047414). The multiple-sequence alignment for amino acid was also performed taking the same NPV isolates as described in nucleotide alignment (Fig. 4). There was no difference found with previously reported HytaNPV, HyinNPV and BusuNPV (Indian Isolate). A difference of 


\begin{tabular}{|c|c|}
\hline $\begin{array}{l}\text { HytaNPV-P } \\
\text { HytaNPV } \\
\text { HyinNPV } \\
\text { BusuNPV (India) } \\
\text { BusuNPV (China) }\end{array}$ & 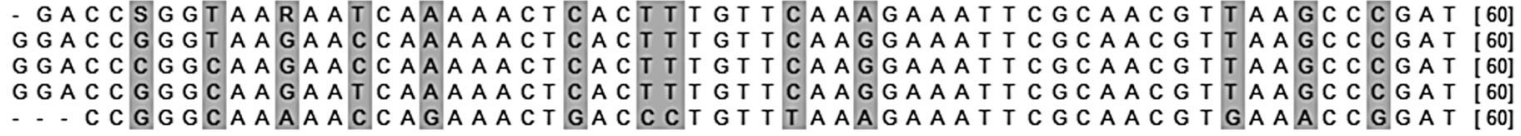 \\
\hline $\begin{array}{l}\text { HytaNPV-P } \\
\text { HytaNPV } \\
\text { HyinNPV } \\
\text { BusuNPV (India) } \\
\text { BusuNPV (China) }\end{array}$ & 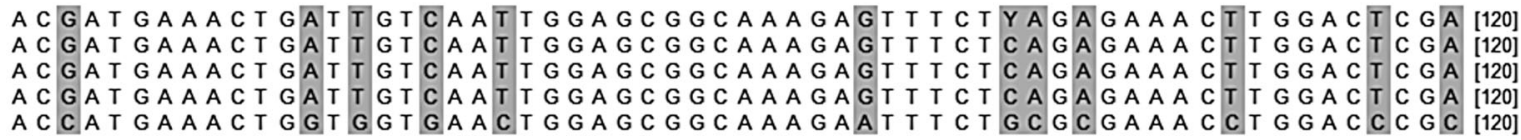 \\
\hline $\begin{array}{l}\text { HytaNPV-P } \\
\text { HytaNPV } \\
\text { HyinNPV } \\
\text { BusuNPV (India) } \\
\text { BusuNPV (China) }\end{array}$ & 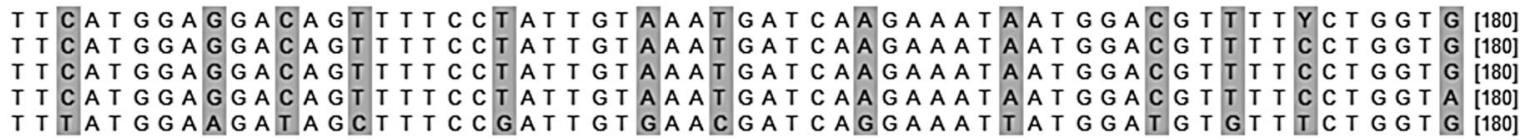 \\
\hline $\begin{array}{l}\text { HytaNPV-P } \\
\text { HytaNPV } \\
\text { HyinNPV } \\
\text { BusuNPV (India) } \\
\text { BusuNPV (China) }\end{array}$ & 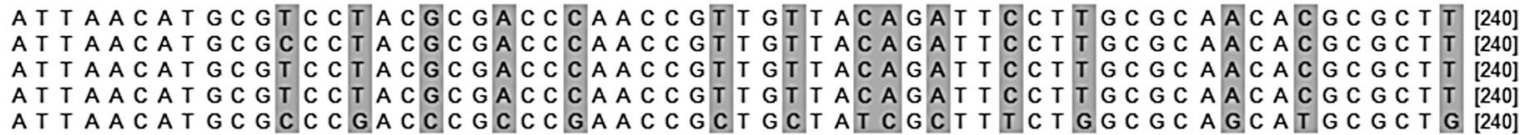 \\
\hline $\begin{array}{l}\text { HytaNPV-P } \\
\text { HytaNPV } \\
\text { HyinNPV } \\
\text { BusuNPV (India) } \\
\text { BusuNPV (China) }\end{array}$ & 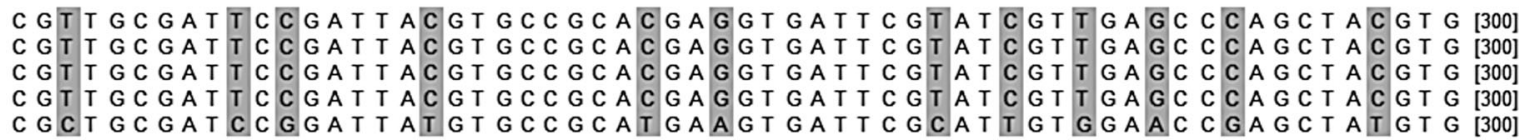 \\
\hline $\begin{array}{l}\text { HytaNPV-P } \\
\text { HytaNPV } \\
\text { HyinNPV } \\
\text { BusuNPV (India) } \\
\text { BusuNPV (China) }\end{array}$ & 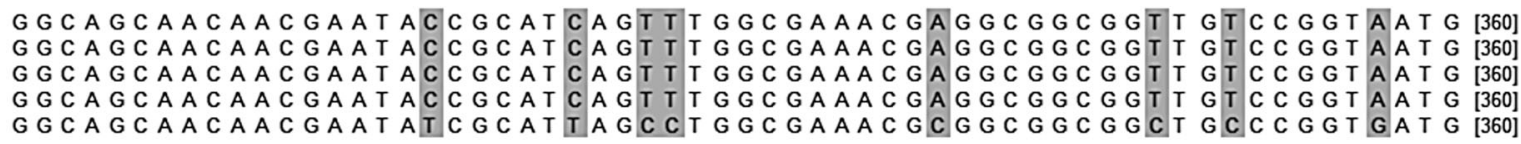 \\
\hline $\begin{array}{l}\text { HytaNPV-P } \\
\text { HytaNPV } \\
\text { HyinNPV } \\
\text { BusuNPV (India) } \\
\text { BusuNPV (China) }\end{array}$ & 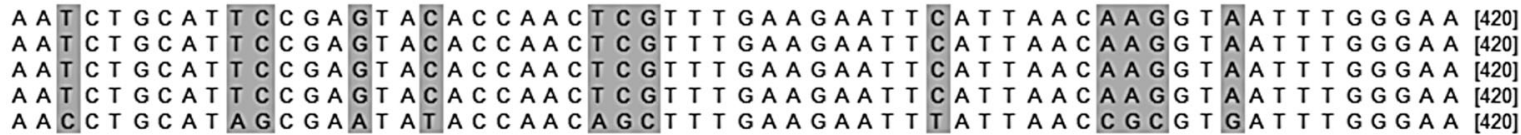 \\
\hline $\begin{array}{l}\text { HytaNPV-P } \\
\text { HytaNPV } \\
\text { HyinNPV } \\
\text { BusuNPV (India) } \\
\text { BusuNPV (China) }\end{array}$ & 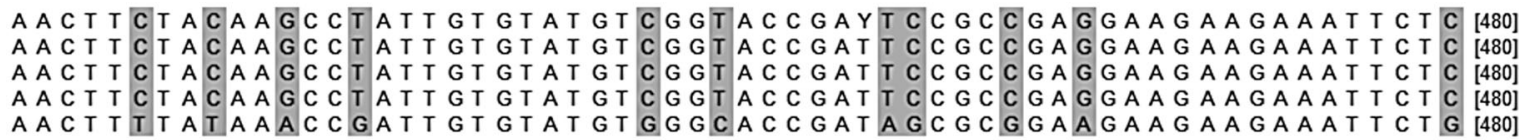 \\
\hline $\begin{array}{l}\text { HytaNPV-P } \\
\text { HytaNPV } \\
\text { HyinNPV } \\
\text { BusuNPV (India) } \\
\text { BusuNPV (China) }\end{array}$ & 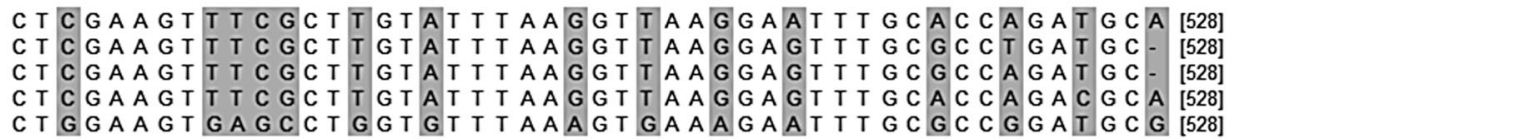 \\
\hline
\end{tabular}

Fig. 3 Comparison of HytaNVP-P polyhedrin gene sequence (acc. no. KP027542) with the sequences of HytaNPV, HyinNPV, BusuNPV (Indian isolate) and BusuNPV (Chinese isolate). Positions containing different nucleotides are shaded

three amino acids (at 87, 146 and 198 positions) was noticed with the BusuNPV of the Chinese isolate.

\section{Phylogenetic analysis}

Phylogenetic analysis of the polyhedrin gene was performed to find out the relationship between HytaNPV-P and other NPVs. The studied data were recognized in the NCBI taxonomy database as a HytaNPV which was referred here as HytaNPV-P. Phylogenetic analysis of the HytaNPV-P polyhedrin showed a high degree of relationship with a large number of published amino acid sequences. A neighbor-joining tree was generated from the amino acid sequences of 26 NPV polyhedrin genes. Phylogenetic analysis indicates that the NPV infecting the $H$. talaca species in the Terai region of India is closely related to HytaNPV, HyinNPV and BusuNPV of the Indian isolate and comes under the same clade (Fig. 5). This suggested that the collected virus-infected larvae from the Terai region were very likely infected by the same strain. It also appeared to have close relation with Chinese isolates of BusuNPV, supported by high bootstrap value. High protein sequence homology of the polyhedrin region of HytaNPV$\mathrm{P}$ with BusuNPV (98\%) of the Chinese isolate and phylogenetic analysis indicates that they are very closely related. Phylogenetic study clearly indicates that in India, a variant of Chinese isolate of BusuNPV infects the H. talaca.

\section{Bioassays}

$\mathrm{LC}_{50}$ and $\mathrm{LT}_{50}$ values were represented in Table 2 for the tested isolates. The mortality ranged from 84 to $22 \%$ and it is clear that $H$. talaca larvae were susceptible to the applied concentrations. The result showed that the second instar 
HytaNPV

HyinNPV

BusuNPV (India)

BusuNPV (China)

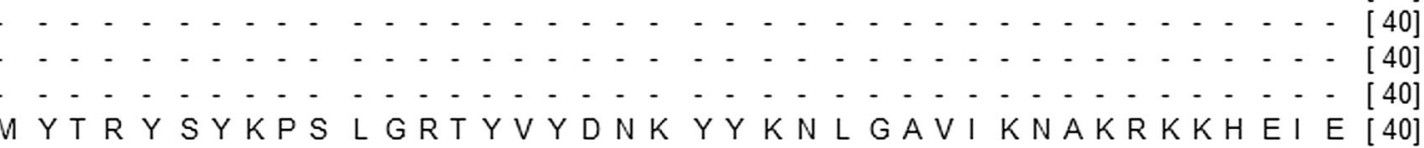

HytaNPV-P

$P G K N Q K L T L F K E I R N V K$ [80]

HytaNPV

HyinNPV

BusuNPV (India)

BusuNPV (China)

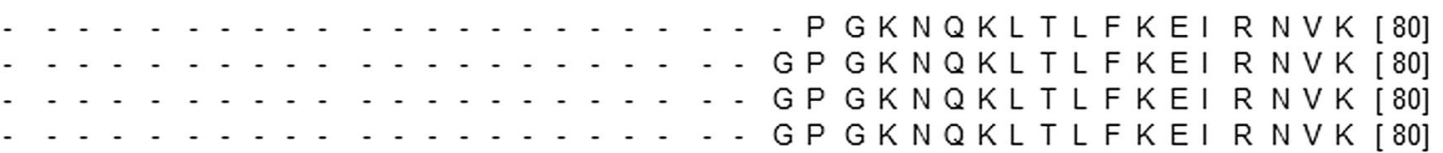

HytaNPV-P

H EVEERTLDPLDKYLVAEDP FL GP GKNQKLTLFKEIRNVK [80]

HytaNPV

HyinNPV

P DTMKLIVNWSGKEFLRETWTR F M D SFPIVNDQEIMDVF [120]

BusuNPV (India)

BusuNPV (China)

$P$ DTMKLI VNWSGKEFLRETWTRFMEDSFPI VNDQEIMDVF

P DTMKLI VNWSGKEFLRETWTRFMEDSFPIVNDQEI M DVF [120]

P DTMKLIVNWSGKEFLRETWTRFMEDSFPI VNDQEI M D TF [120]

P DTMKLVVNWSGKEFLRETWTRFMEDSFPI VNDQEIM DVF [120]

$\begin{array}{llll}\text { HytaNPV-P } & L\end{array}$

HytaNPV

HyinNPV

L VINMRPTRP NRCYRFLA Q A ALRC DSDYVPHEVIRIVEPS [160]

BusuNPV (India)

BusuNPV (China)

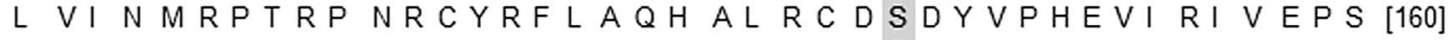

L VI N MRPTRP NRCYRFLAQH ALRCDSDYVPHEVIRIVEPS [160]

HytaNPV-P

HytaNPV

HyinNPV

L VINMRPTRP NRCYRFLAQH ALR C DPDYVPHEVIRIVEPS [160]

BusuNPV (India)

BusuNPV (China)

YVGSN NEYRI SLAKRGGGCP VMNLHSEYTNSFEEFIN KVI

Y VGSN NEYRI SLAKRGGGCP VMNLHSEYTNSFEEFI N KVI

$Y V G S N N E Y R I S L A K R G G G C P V M N L H S E Y T N S F E E F I N K V I$

$Y$ VGSN NEYRI SLAKRGGGCP VMNLHSEYTNSFEEFINKVI

[200]

$Y V G S N N E Y R I S L A K R G G G C P V M N L H S E Y T N S F E E F I N R V I$

HytaNPV-P

HytaNPV

HyinNPV

WENFYKPIVYVGTDSAEEEE I L L E V L L K K V KEFAPDA- - [240] WENF Y KPI VY VGTDSAEEEEI L L E V L VFKVKEFAPDA- - [240] WENF Y KPI VY VGTDSAEEEEI L L E V L VFKVKEFAPDA- - [240]

BusuNPV (India)

BusuNPV (China)

WENFYKPIVYVGTDSAEEEE I L LEVSLVFKVKEFAPDA- - [240] WENFYKPIVYVGTDSAEEEE I L L E V L VFKVKEFAPDAPL [240]

\begin{tabular}{|c|c|c|c|c|c|}
\hline Hytal & - & - & . & & {$[24$} \\
\hline HytaNPV & - & - & . & & [246] \\
\hline Hyi & . & - & . & & 24 \\
\hline טר & - & - & - & & {$[24$} \\
\hline SusuNPV (China) & $Y T$ & $G P$ & A & $Y$ & {$[24$} \\
\hline
\end{tabular}

Fig. 4 Deduced amino acid sequence alignment of HytaNPV-P poyhedrin gene (acc. no. AJN00735) with HytaNPV (acc. no. AEK86285), HyinNPV (acc. no. AEK86286), BusuNPV (Indian

was most susceptible to $\mathrm{SMB}$ isolate with an $\mathrm{LC}_{50}$ of $8.15 \times 10^{4} \mathrm{POBs} / \mathrm{ml}$. Larvae were also susceptible to infection with $\mathrm{LC}_{50}$ values of $3.87 \times 10^{5}, 1.89 \times 10^{5}$ and $2.79 \times 10^{5} \mathrm{POBs} / \mathrm{ml}$, for RJB, WSB and TLP isolates, respectively, within the same time frame. The results of the study reflected the dose-dependent mortality, with the rising dose of POBs, the larval mortality showed an increasing trend. Inoculated larvae started to die at second day post-infection. In untreated control, no mortality was found. The estimated $\mathrm{LT}_{50}$ values varied from 3.25 to 6.67 days. An instance, for highest concentration $\left(1 \times 10^{8} \mathrm{POBs} / \mathrm{ml}\right)$ of the $\mathrm{LT}_{50}$ value of the WSB isolate was lowest at 3.25 days. When treated with the highest dose, the $\mathrm{LT}_{50}$ values were 3.5 days for the SMB isolate, isolate, acc. no. AEK86284) and BusuNPV (Chinese isolate, acc. no. YP009001778). Positions containing different amino acids are shaded

3.86 days for the RJB isolate and 3.89 days for the TLP isolate. On the other hand, the result also indicates a gradual increase in tolerance with lower concentration.

\section{Discussion}

Virus is now an important subject for the development of biopesticides and to maintain the ecosystem in the agricultural field. Search of a new natural isolate of virus, which contains better biopesticidal characteristics, to control insect pest is still an important aspect in biological control. In this regard, baculoviruses like NPVs are well known for their variability, frequent genetic variation and 
Fig. 5 Phylogenetic analysis using the predicted amino acid residues from Hyposidra talaca NPV (HytaNPV-P) polyhedrin gene. The tree shows the relationship between HytaNPV$\mathrm{P}$ and other NPVs. The tree was constructed by neighbor-joining algorithm using MEGA (v. 6.06). The branch numbers represent bootstrap probabilities of 1000 replicates. The scale bar represents 0.05 amino acid substitutions per site. The tree is rooted on the Bombyx mori NPV for comparison. Nodes with bootstrap value above $50 \%$ are shown. The analysis involved $26 \mathrm{NPV}$ amino acid sequences; the position of HytaNPV-P polyhedrin is highlighted (in red diamond). All positions containing gaps and missing data were eliminated

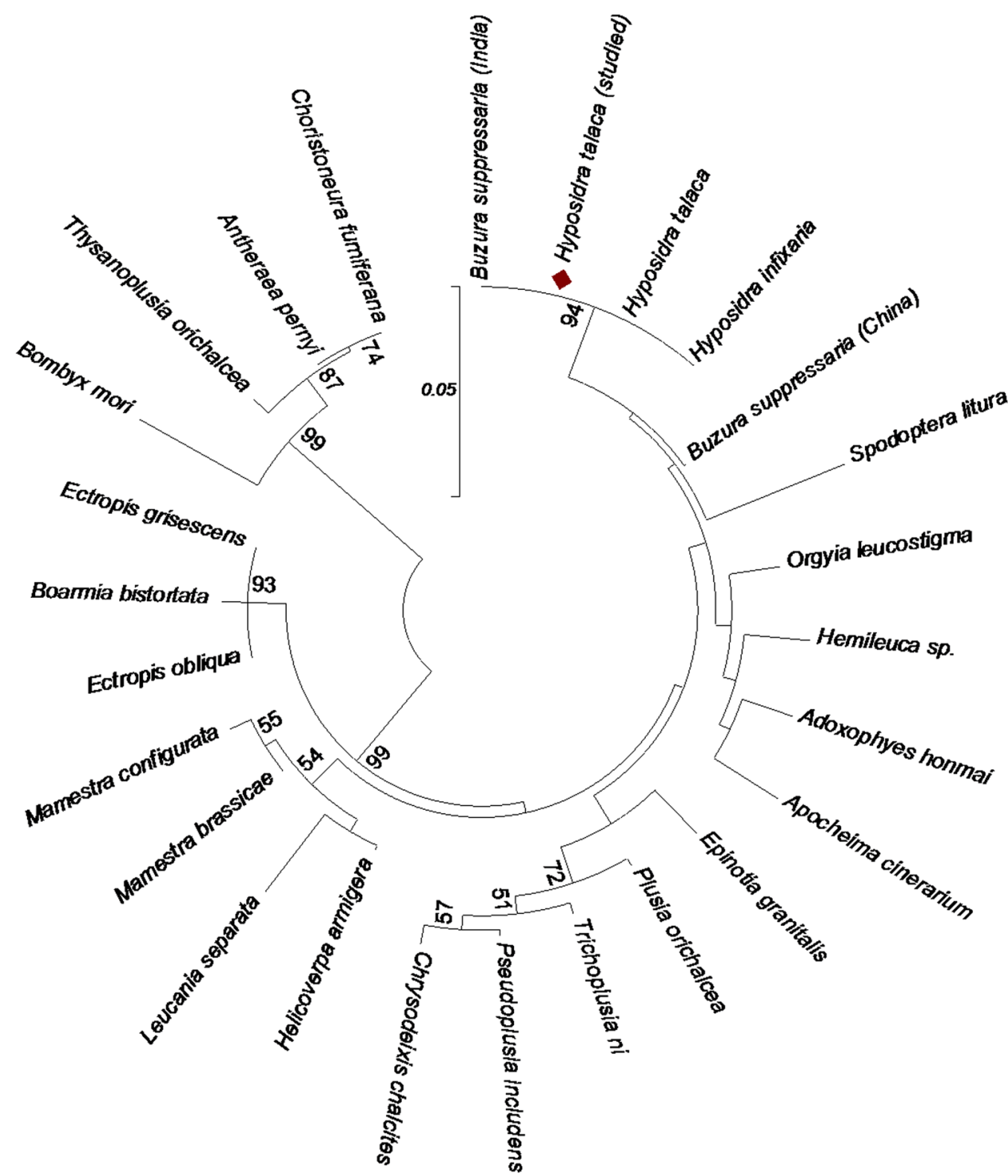

differences in their biology. These qualities remain intact even when the NPV isolates are sampled from the same species of different geographical locations (Cory et al. 2005).

H. talaca plays a leading role in reducing tea production throughout the north-eastern tea hub of India (Basu Majumdar and Ghosh 2004). H. talaca was reported as a tea defoliator in Java (Yunus and Ho 1980) and it was also reported as a major pest of mango, cacao and other forest trees (Entwistle 1972; Muniappan and Viraktamath 1986; Singh and Singh 2004). Some entomopathogenic bacteria and NPV, infecting the pest, have been reported from north-eastern part of India (Mukhopadhyay et al. 2010; Antony et al. 2011). However, commercial use of these microorganisms in this region is still far away. Unlike China and Japan, there is no considerable progress in using naturally occurring baculoviruses in integrated pest management of tea in India. In China, significant response of BusuNPV and EcobNPV against B. suppressaria and $E$. oblique made these NPVs commercially available (Hu et al. 1993; Ma et al. 2007). In India, HytaNPV can be an effective control measure against $H$. talaca. The result of the PCR study showed the presence of single NPV strain across the entire north-eastern tea hub of India. In lepidopteran NPVs, the full length of polyhedrin gene ranges from 483 to $747 \mathrm{bp}$ (Jehle et al. 2006), and the $527 \mathrm{bp}$ partial segment of the studied polyhedrin gene also fits within it. In the present study, the partial amplification of HytaNPV-P infecting $H$. talaca, showed $98 \%$ nucleotide sequence identity and $100 \%$ similarity in amino acids with previously published HytaNPV (Antony et al. 2011). HytaNPV-P also showed the same sequence identity with HyinNPV and BusuNPV (Indian isolate). This implies that HytaNPV-P is closely related to the aforesaid NPVs. 
Table 2 Dose-mortality response of second instar Hyposidra talaca larvae on four geographic isolates of $H$. talaca nucleopolyhedrovirus (HytaNPV) within 7 days of inoculation

\begin{tabular}{|c|c|c|c|c|c|c|c|}
\hline $\begin{array}{l}\text { HytaNPV } \\
\text { isolate }\end{array}$ & $\begin{array}{l}\text { No. of tested 2nd instar } \\
\text { larvae }(n)\end{array}$ & $\begin{array}{l}\text { Concentration } \\
\text { (POBs/ml) }\end{array}$ & $\begin{array}{l}\text { HytaNPV caused } \\
\text { mortality }(\%)\end{array}$ & Slope \pm SE & $\begin{array}{l}\mathrm{LC}_{50} \mathrm{POBs} / \mathrm{ml}(95 \% \\
\text { confidence limits) }\end{array}$ & $\begin{array}{l}\text { Chi } \\
\text { square } \\
\left(\chi^{2}\right)\end{array}$ & $\begin{array}{l}\mathrm{LT}_{50} \\
\text { (days) }\end{array}$ \\
\hline \multirow[t]{7}{*}{ SMB } & 90 & $1 \times 10^{8}$ & 84 & \multirow[t]{7}{*}{$0.332 \pm 0.035$} & \multirow{7}{*}{$\begin{array}{l}8.15 \times 10^{4}\left(3.39 \times 10^{4}-\right. \\
\left.1.78 \times 10^{5}\right)\end{array}$} & \multirow[t]{7}{*}{0.097} & 3.50 \\
\hline & 90 & $1 \times 10^{7}$ & 77 & & & & 4.27 \\
\hline & 90 & $1 \times 10^{6}$ & 63 & & & & 5.14 \\
\hline & 90 & $1 \times 10^{5}$ & 51 & & & & 6.67 \\
\hline & 90 & $1 \times 10^{4}$ & 38 & & & & - \\
\hline & 90 & $1 \times 10^{3}$ & 27 & & & & - \\
\hline & 90 & Control & 0 & & & & - \\
\hline \multirow[t]{7}{*}{ RJB } & 90 & $1 \times 10^{8}$ & 77 & \multirow[t]{7}{*}{$0.297 \pm 0.034$} & \multirow{7}{*}{$\begin{array}{l}3.87 \times 10^{5}\left(1.62 \times 10^{5}-\right. \\
\left.\quad 9.44 \times 10^{5}\right)\end{array}$} & \multirow[t]{7}{*}{0.108} & 3.86 \\
\hline & 90 & $1 \times 10^{7}$ & 67 & & & & 4.80 \\
\hline & 90 & $1 \times 10^{6}$ & 53 & & & & 5.80 \\
\hline & 90 & $1 \times 10^{5}$ & 43 & & & & - \\
\hline & 90 & $1 \times 10^{4}$ & 32 & & & & - \\
\hline & 90 & $1 \times 10^{3}$ & 22 & & & & - \\
\hline & 90 & Control & 0 & & & & - \\
\hline \multirow[t]{7}{*}{ WSB } & 90 & $1 \times 10^{8}$ & 83 & \multirow[t]{7}{*}{$0.350 \pm 0.035$} & \multirow{7}{*}{$\begin{array}{l}1.89 \times 10^{5}\left(8.73 \times 10^{4}-\right. \\
\left.\quad 3.99 \times 10^{5}\right)\end{array}$} & \multirow[t]{7}{*}{0.375} & 3.25 \\
\hline & 90 & $1 \times 10^{7}$ & 73 & & & & 4.20 \\
\hline & 90 & $1 \times 10^{6}$ & 60 & & & & 5.33 \\
\hline & 90 & $1 \times 10^{5}$ & 43 & & & & - \\
\hline & 90 & $1 \times 10^{4}$ & 33 & & & & - \\
\hline & 90 & $1 \times 10^{3}$ & 22 & & & & - \\
\hline & 90 & Control & 0 & & & & - \\
\hline \multirow[t]{7}{*}{ TLP } & 90 & $1 \times 10^{8}$ & 78 & \multirow[t]{7}{*}{$0.300 \pm 0.034$} & \multirow{7}{*}{$\begin{array}{l}2.79 \times 10^{5}\left(1.17 \times 10^{5}-\right. \\
\left.\quad 6.65 \times 10^{5}\right)\end{array}$} & \multirow[t]{7}{*}{0.081} & 3.89 \\
\hline & 90 & $1 \times 10^{7}$ & 69 & & & & 4.75 \\
\hline & 90 & $1 \times 10^{6}$ & 56 & & & & 5.86 \\
\hline & 90 & $1 \times 10^{5}$ & 45 & & & & - \\
\hline & 90 & $1 \times 10^{4}$ & 33 & & & & - \\
\hline & 90 & $1 \times 10^{3}$ & 23 & & & & - \\
\hline & 90 & Control & 0 & & & & - \\
\hline
\end{tabular}

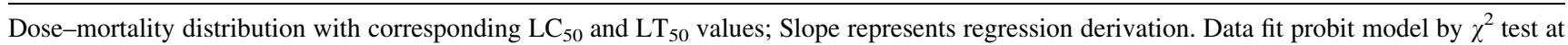
$\alpha=0.05$, degree of freedom $(d f)=5$

Interestingly, HytaNPV-P also showed high sequence identity with the Chinese isolate of NPV infecting $B$. suppressaria. In this case, HytaNPV-P showed 98\% similarity in amino acids with BusuNPV (acc. no. YP009001778, Chinese isolate) and only three amino acid differences were observed in the polyhedrin gene of HytaNPV-P and BusuNPV (Chinese isolate). This similarity indicates that HytaNPV-P is also related to the isolate of BusuNPV from China.

Phylogenetic studies have shown that HytaNPV-P, HytaNPV, HyinNPV and BusuNPV (Indian isolate) belong to same clade and the derived tree also encourages the fact that a variant of the Chinese isolate of BusuNPV (acc. no. YP009001778) infects the HytaNVP-P. However, full genome sequencing is necessary to confirm whether the
HytaNPV-P altogether is a different virus or a different isolate of BusuNPV (China).

After ingestion of polyhedral occlusion bodies by larvae, NPV virions reach the midgut and spread to cause infection (Petrik et al. 2003). The results showed the effectiveness of the application of isolated NPV through tea leaves. In the present study, the SMB isolate of the Terai region showed higher $\mathrm{LC}_{50}$ value for second instar than the previously reported value of $2.8 \times 10^{3} \mathrm{POBs} / \mathrm{ml}$ (from Terai region) by Mukhopadhyay et al. (2011). On the other hand, we found the RJB isolate (from Eastern Dooars), WSB isolate (from Western Dooars) and TLP isolate (from Central Dooars), which were respectively 5 times, 2.3 times and 3.4 times less active than the SMB isolate. The reasons for the variability may be the different geographic locations 
(Terai and Dooars) and differences in larval age (Payne et al. 1981) and feeding habit (Lacey et al. 2002). The number of virions per occlusion body and the host susceptibility differences to NPV (El-Salamouny et al. 2003) can be the other possible causes. However, for cross reference, there is no other report of bioassay available at this point for this particular tea pest. Stiles and Himmerich (1998) also observed higher $\mathrm{LC}_{50}$ of AcMNPV against second instar Heliothis zea (i.e., $6.38 \times 10^{5} \mathrm{POBs} / \mathrm{ml}$ ). Second instar larvae of Spodoptera littoralis were also highly susceptible to the SpliMNPV at the higher concentration of $2 \times 10^{6} \mathrm{POBs} / \mathrm{ml}$, which caused a high rate of mortality than the lower concentration (Sutanto et al. 2014).

The virulence of baculoviruses is measured by the killing speed of the concerned virus to the insect host (VanBeek and Hughes 1998) and is necessary to know the correct time for pest management programs. The highest inoculums of $10^{8} \mathrm{POBs} / \mathrm{ml}$ of HytaNPV showed rapid killing speed with $\mathrm{LT}_{50}$ value of 3.25 to 3.89 days after treatment for four isolates. Lower concentrations take longer time to meet the same level of larval mortality.

The result shows decreasing trend in mortality with the decreasing dosage. Although this relation between the rate of change in mortality with the lessening dosage is not directly proportional, as the dose decreases ten times the mortality rate is decreased well less than ten times. Previous studies also support this observation (Jankevica and Zarins 1999; Sajap et al. 2000; Sethuraman and Narayanan 2010; Mukhopadhyay et al. 2011; Sutanto et al. 2014). However, the regression result in Table 2 shows that a positive correlation exists between mortality and dosage, as all the slope values are positive and infinitesimal Chi squares signify that there is a good linear fit. Both the studies of $\mathrm{LC}_{50}$ and $\mathrm{LT}_{50}$ satisfied the insecticidal characteristics of HytaNPV. HytaNPV isolated from $H$. talaca is being developed as a biopesticide for the control of $H$. talaca.

\section{Conclusion}

In this paper, we described the cloning and sequencing of a highly conserved region of polyhedrin gene and the insecticidal activity of HytaNPV. The PCR study revealed the presence of the same viral strain throughout the northeastern tea belt. The high degree of phylogenetic agreement between the studied and previously reported HytaNPV polyhedrin gene sequence also supports this inference. This is the first report of sequencing of HytaNPV polyhedrin gene from the Terai region. The availability of HytaNPV may help in biopesticide production. The obtained results of bioassays support the use of HytaNPV as a potent biological control agent and a competent alternative to chemical insecticides in integrated pest management (IPM). The aforementioned characterization of the newly isolated HytaNPV would provide better understanding of the molecular properties of this virus and be helpful in the development of HytaNPV as a biopesticide.

Acknowledgements The molecular work was performed at the Department of Biotechnology and Bioinformatics Cell, Plant Improvement Division, Tea Research Association, Tocklai Tea Research Institute. The bioassay part was supported by the North Bengal Regional Research and Development Centre, Tea Research Association. The authors wish to thank Picklu Mandal for all his assistance during sample collection. We also thank the managers of all the participated tea plantations for their cooperation and support.

\section{Compliance with ethical standards}

Disclosure The sequence reported in this paper has been deposited in NCBI under accession no. KP027542. No potential conflict of interest was reported by the authors.

Funding This work was supported by the Tea Research Association, Tocklai Tea Research Institute, India.

Open Access This article is distributed under the terms of the Creative Commons Attribution 4.0 International License (http:// creativecommons.org/licenses/by/4.0/), which permits unrestricted use, distribution, and reproduction in any medium, provided you give appropriate credit to the original author(s) and the source, provide a link to the Creative Commons license, and indicate if changes were made.

\section{References}

Antony B, Sinu PA, Das S (2011) New record of nucleopolyhedroviruses in tea looper caterpillars in India. J Invertebr Pathol 108:63-67

Arneodo J, König G, Berretta M, Di Rienzo J, Taboga O, ScioccoCap A (2012) Detection and kinetic analysis of Epinotia aporema granulovirus in its lepidopteran host by real-time PCR. Arch Virol 157(3):405-409

Basu Majumdar A, Ghosh P (2004) Hyposidra talaca (Walker) a destructive pest of tea in Dooars tea plantations. Two Bud 51:49-51

Biever KD, Hostetter DL (1971) Activity of nuclear polyhedrosis virus of the cabbage looper evaluated at programmed temperature regimes. J Invertebr Pathol 18:81-84

Burand J, Horton H, Retnasami S, Elkington J (1992) The use of polymerase chain reaction and shortwave UV irradiation to detect baculovirus DNA on the surface of gypsy moth eggs. J Virol Methods 36:141-150

Cory JS, Green BM, Paul RK, Hunter-Fujita F (2005) Genotypic and phenotypic diversity of a baculovirus population within an individual insect host. J Invertebr Pathol 89:101-111

El-Salamouny S, Lange M, Jutzi M, Huber J, Jehle JA (2003) Comparative study on the susceptibility of cutworms (Lepidoptera: Noctuidae) to Agrotis segetum nucleopolyhedrovirus and Agrotis ipsilon nucleopolyhedrovirus. J Invertebr Pathol 84:75-82

Entwistle PF (1972) Pests of Cacao. Longmans, London, p 779 
Fattouch S, Acheche H, M'hirsi S, Mellouli L, Bejar S, Marrakchi M, Marzouki N (2005) RT-PCR-RFLP for genetic diversity analysis of Tunisian Grapevine Fanleaf virus isolates in their natural host plants. J Virol Methods 127:126-132

Galal F (2009) Universal primer for early and rapid detection of nucleopolyhedroviruses of multiple species using polymerase chain reaction. Egypt Acad J Biol Sci 1(1):57-64

Hazarika LK, Bhuyan M, Hazarika MN (2009) Insect pests of tea and their management. Annu Rev Entomol 54:267-284

Hewson I, Brown J, Gitlin S, Doud D (2011) Nucleopolyhedrovirus detection and distribution in terrestrial, freshwater, and marine habitats of Appledore Island, Gulf of Maine. Microb Ecol 62(1):48-57

Hu ZH, Liu MF, Jin F, Wang ZH, Liu XY, Li MJ, Liang BF, Xie TE (1993) Nucleotide sequence of the Buzura suppressaria single nuclear polyhedrosis virus polyhedrin gene. J Gen Virol 74:1617-1620

Ikuno A, Margatho L, Harakava R, Akamatsu M, Martins E, Porto A, Ferreira V (2004) Direct application of the new PCR protocol for evaluation and monitoring of Bombyx mori infection by nucleopolyhedrovirus. Arq Inst Biol 71(3):309-315

Jankevica L, Zarins I (1999) Virulence of Malacosoma neustria nucleopolyhedrovirus Latvian isolates. Latv Entomol 37:40-45

Jehle J (2004) The mosaic structure of the polyhedrin gene of the Autographa californica Nucleopolyhedrovirus (AcMNPV). Virus Genes 29(1):5-8

Jehle J, Blissard G, Bonning B, Cory J, Herniou E, Rohrmann G, Theilmann D, Thiem S, Vlak J (2006) On the classification and nomenclature of baculoviruses: a proposal for revision. Arch Virol 151:1257-1266

Lacey LA, Vail PV, Hoffmann DF (2002) Comparative activity of baculoviruses against the codling moth Cydia pomonella and three other tortricid pests of tree fruit. J Invertebr Pathol 80:64-68

Ma XC, Shang JY, Yang ZN, Bao YY, Xiao Q, Zhang CX (2007) Genome sequence and organization of a nucleopolyhedrovirus that infects the Tea Looper Caterpillar, Ectropis oblique. Virology 360:235-246

Moraes R, Maruniak J (1997) Detection and identification of multiple baculoviruses using the polymerase chain reaction (PCR) and restriction endonuclease analysis. Mol Cells 11:334-340

Moscardi F (1999) Assessment of the application of baculoviruses for control of Lepidoptera. Annu Rev Entomol 44:257-289

Mukhopadhyay A, De D, Khewa S (2010) Exploring the biocontrol potential of naturally occurring bacterial and viral entomopathogens of defoliating lepidopteran pests of tea plantations. J Biopestic 3:117-120

Mukhopadhyay A, Khewa S, De D (2011) Characteristics and virulence of nucleopolyhedrovirus isolated from Hyposidra talaca (Lepidoptera: Geometridae), a pest of tea in Darjeeling Terai, India. Int J Trop Insect Sci 31(1-2):13-19

Muniappan R, Viraktamath CA (1986) Insects and mites associated with Chromolaena odorata (L.) R. M. King and H. Robinson (Asteraceae) in Karnataka and Tamil Nadu. Entomon 11:285-287

Payne CC, Tatchell GM, Williams CF (1981) The comparative susceptibilities of Pieris brassicae and Pieris rapae to a granulosis virus from Pieris brassicae. J Invertebr Pathol $38: 273-280$
Petrik DT, Iseli A, Montelone BA, Van Etten JL, Clem RJ (2003) Improving baculovirus resistance to UV inactivation: increased virulence resulting from expression of a DNA repair enzyme. J Invertebr Pathol 82(1):50-56

Saitou N, Nei M (1987) The neighbor-joining method: a new method for reconstructing phylogenetic trees. Mol Bol Evol 4(4):406-425

Sajap AS, Kotulai JR, Bakir MA, Hong LW, Samad MA, Kadir HA (2000) Pathogenicity and characteristics of Spodoptera litura nucleopolyhedrovirus from Peninsular Malaysia. Pertan J Trop Agric Sci 23(1):23-28

Sannigrahi S, Talukdar T (2003) Pesticide use patterns in Dooars tea industry. Two Bud 50:35-38

Sarker M, Mukhopadhyay A (2006) A preliminary study on host plant related changes of general esterase in Buzura suppressaria (Lepidoptera: Geometridae), a major defoliator of tea in the Darjeeling foothills and adjoining plains in India. Sri Lanka J Tea Sci 71:1-7

Sethuraman V, Narayanan K (2010) Biological activity of Nucleopolyhedrovirus Isolated from Chilo partellus (Swinhoe) (Lepidoptera: Pyralidae) in India. Asian J Exp Biol Sci 1(2):325-330

Singh DP, Singh S (2004) Hyposidra talaca walk. (Lepidoptera: Geometridae) a new pest of Quercus incana Roxb. Indian For 130:231-232

Sinu PA, Mandal P, Antony B (2011) Range expansion of Hyposidra talaca (Geometridae: Lepidoptera), a major pest, to Northeastern Indian tea plantations: change of weather and anti-predatory behaviour of the pest as possible causes. Int $\mathbf{J}$ Trop Insect Sci 3548:1-7

Sinu PA, Talukdar T, Mallick S, Mandal P, Dasgupta S (2015) In situ mortality of Hyposidra talaca (Geometridae: Lepidoptera) by its nucleopolyhedrovirus and comparison of tea production in untreated and chemical insecticide-treated plots. Biocontrol Sci Technol 25(3):352-358

Stiles B, Himmerich S (1998) Autographa californica NPV isolates: Restriction endonuclease analysis and comparative biological activity. J Invertebr Pathol 72:174-177

Sutanto KD, El-Salamouny S, Al-Dawood AS (2014) Affectivity of Spodoptera littoralis nucleopolyhedrovirus (SpliNPV) against first and second instar larvae of the cotton leafworm, Spodoptera littoralis (Boisd.) (Lepidoptera: Noctuidae). Afr J Microbiol Res 8(4):337-340

Tamura K, Stecher G, Peterson D, Filipski A, Kumar S (2013) MEGA6: molecular evolutionary genetics analysis version 6.0. Mol Biol Evol 30(12):2725-2729

Thompson JD, Higgins DG, Gibson TJ (1994) CLUSTAL W: improving the sensitivity of progressive multiple sequence alignment through sequence weighting, position specific gap penalties and weight matrix choice. Nucl Acids Res 22:4673-4680

Van-Beek NA, Hughes PR (1998) The response time of insect larvae infected with recombinant bacuoloviuses. J Invertebr Pathol $72: 338-347$

Woo SD (2001) Rapid detection of multiple nucleopolyhedroviruses using polymerase chain reaction. Mol Cells 11:334-340

Yunus A, Ho TH (1980) List of economic pests, host plants, parasites and predators in West Malaysia (1920-1978). Bull Malays Dep Agric 153:538 